\title{
About the Editor
}

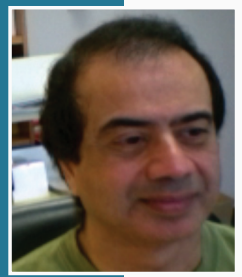

Jaffer A Ajani

Jaffer A Ajani is Professor of Medicine and an internist at the University of Texas MD Anderson Cancer Center (TX, USA). His main interest is in gastrointestinal oncology research and education. He is board-certified in family medicine and internal medicine, as well as in the medical oncology subspecialty. After completing his undergraduate studies with distinction in applied physics, he went on to earn his MD degree from Government Medical College in Nagpur, India. He remained at the college to complete a rotating internship and a residency in general surgery and orthopedics before continuing on to Pennsylvania State University (PA, USA) for internship and residency training in family practice. He then completed an internship and residency in internal medicine at Tulane University School of Medicine (LA, USA) and was subsequently awarded a clinical fellowship in medical oncology at the MD Anderson Cancer Center (TX, USA). He remained at the Center for an additional 2-year research fellowship in medical oncology. He has more than 300 peerreviewed publications. He is the Founder of the International Society of Gastrointestinal Oncology and Editor-in-Chief of a new journal called Gastrointestinal Cancer Research.

For reprint orders, please contact: reprints@futuremedicine.com 


\section{Foreword}

\section{Gastroesophageal cancers: providing an update}

\section{Jaffer A Ajani}

Many years of diligent efforts by a variety of investigators from all over the world have culminated into progress that is now palpable. We can point to many trials that have changed the standard of care for patients with gastroesophageal cancer. I am pleased to introduce five chapters in this book, written by experts in the field. It is my hope that these chapters will provide rapid access to information you need to improve your general knowledge about gastroesophageal cancer and to improve care of your patients. I would like to briefly summarize each of the five chapters as follows.

In Chapter 1, Roy, Cunningham and Chau discuss epidemiology of gastric cancer and racial disparity, etiology, including the role of Helicobacter pylori, various histology phenotypes and provide some discussion of molecular biology. This chapter is written by highly experienced investigators and their insights are appreciated. It appears clear that several populations are highly susceptible to gastric tumorigenesis but also considerably influenced by environmental factors. We have the opportunity to influence our environmental factors to reduce the risk of the development of gastric carcinoma.

Moehler, Palmqvist, Lang, Meyer and Gockel discuss surgical approaches for gastric cancer in Chapter 2. This stellar group of surgeons emphasizes high-volume centers and high-volume surgeons, as well as surgery with a more elaborate dissection of lymph nodes (D2 dissection). Although there 
are controversies surrounding the recommendation of D2 dissection, it is clear that a D2 dissection provides proper nodal staging and a low level of complications in experienced hands.

In Chapter 3, Catalano and Graziano discuss the current approaches to localized gastric carcinoma. These two highly experienced investigators provide concise information on pre- and post-operative approaches. Considerable accumulation of level 1 evidence for the treatment of localized gastric cancers is heartening. Clearly, the next era will bring more individualized approaches to these tumors based on tumor biology and patient genetics.

Ilson and Smyth tackles the issue of target therapy or biologic therapy in Chapter 4. The author brings a world of experience in writing this chapter. This is a rapidly developing arena and will be driven by increasing understanding of molecular pathways that drive these tumors. Although the outcome of the AVAGAST study is not favorable, many lessons can be learned from it. We have learned that clinical practices in treating patients with gastric cancer vary considerably and some of these differences are multifactorial. However, we are also learning that biologic differences among populations could be driving the response to certain biologic agents and this knowledge will be the incentive for developing effective therapies in the future.

In Chapter 5, Ghai and Jiang provide an overview on the management of gastric cancer to include published guidelines and insights into how to interpret trials incorporating surgical questions and preoperative and postoperative strategies. In addition, they provide an update on important studies presented at the American Socitey of Clinical Oncology 2011. Their invaluable contribution is appreciated.

I acknowledge that at this stage, this book does not cover all aspect of gastroesophageal cancer, but we have focused on essential themes and hope to build on it in the future.

Financial \& competing interests disclosure

The author has no relevant affiliations or financial involvement with any organization or entity with a financial interest in or financial conflict with the subject matter or materials discussed in the manuscript. This includes employment, consultancies, honoraria, stock ownership or options, expert testimony, grants or patents received or pending, or royalties.

No writing assistance was utilized in the production of this manuscript. 